\section{Leonardo Santana Viloria*}

Universidad de Bogotá Jorge Tadeo Lozano, Bogotá, Colombia.

Recibido: 20 de agosto de 2014

Concepto de evaluación: 25 de noviembre de 2014

Aprobado: 27 de noviembre de 2014

Artículo de investigación (C) 2015 Universidad Católica de Colombia. Facultad de Ciencias Económicas y Administrativas. Todos los derechos reservados

* Magíster en Ciencias Económicas y economista. Docente asociado de la Universidad de Bogotá Jorge Tadeo Lozano, Bogotá, Colombia. Forma parte del Grupo de Estudios en Teoría Financiera e Inversiones (Getfin). Dirección de correspondencia: Cra. 4 N. ${ }^{2} 22-61$, of. 534, módulo 1.Correo electrónico: leonardo.santana@utadeo.edu.co
Finanz. polit. econ., ISSN: 2248-6046, Vol. 7, No. 1, enero-junio, 2015, pp. 83-95 http://dx.doi.org/10.14718/revfinanzpolitecon.2015.7.1.4

\section{Estimación del beta para el sector inmobiliario a partir del desempeño de fondos de inversión inmobiliaria en Colombia ${ }^{1}$}

\section{RESUMEN}

La creación de fondos de inversión inmobiliaria en Colombia ha abierto posibilidades de diversificación de portafolio a agentes que deseen invertir en el sector inmobiliario sin tener que comprar y administrar finca raíz de forma directa. El comportamiento de estos fondos ha mostrado una rentabilidad promedio superior y una volatilidad menor que la del mercado durante los últimos años. Este artículo aplica el modelo de valoración de activos de capital (CAPM) y estima varios modelos autorregresivos y de heterocedasticidad condicional, con el fin de calcular el beta de estos fondos como una aproximación a la sensibilidad al riesgo sistemático del sector inmobiliario. Los resultados de las estimaciones muestran que el nivel de riesgo del sector inmobiliario se encuentra muy por debajo del riesgo de mercado, lo cual sugiere que los proyectos inmobiliarios tienen un costo de capital mucho menor que el de determinados proyectos en otros sectores.

Palabras clave: fondos de inversión inmobiliaria, sector inmobiliario, modelo CAPM, modelos GARCH.

JEL: C20, D81, E22, F21, G11, G32

\section{Estimating Beta for the Real Estate Sector Based on the Real Estate Investment Trusts Performance in Colombia}

\section{ABSTRACT}

The creation of property investment funds in Colombia has made portfolio diversification possible by allowing parties to invest in the property sector without buying and managing real estate directly. In recent years, the behavior

1 Este artículo es resultado del proyecto de investigación "Análisis de los fondos de inversión inmobiliaria en Colombia", financiado por la Universidad de Bogotá Jorge Tadeo Lozano, durante el año 2012. 
of these funds has shown higher average profits and lower volatility than that of the market. This study applies the Capital Asset Pricing Model (CAPM) and estimates various autoregressive models and models of conditional heteroscedasticity, in order to calculate the beta of these funds as an estimate of the property sector's sensitivity to systematic risk. Results show that the risk level of the property sector is far lower than that of the market, suggesting that real estate projects have much lower capital costs than projects in other sectors.

Keywords: property investment funds, property sector, CAPM model, GARCH models.

\section{Estimação do beta para o setor imobiliário a partir do desempenho de fundos de investimento imobiliário na Colômbia}

\section{RESUMO}

A criação de fundos de investimento imobiliário na Colômbia abriu possibilidades de diversificação de carteira de títulos a agentes que desejarem investir no setor imobiliário sem ter que comprar e administrar compra e venda de imóveis de forma direta. O comportamento desses fundos tem mostrado uma rentabilidade média superior e uma volatilidade menor que a do mercado durante os últimos anos. Este artigo aplica o modelo de avaliação de ativos financeiros (CAPM) e estima vários modelos autorregressivos e de heterocedasticidade condicional a fim de calcular o beta desses fundos com uma aproximação à sensibilidade ao risco sistemático do setor imobiliário. Os resultados das estimações mostram que o nível de risco do setor imobiliário se encontra muito abaixo do risco de mercado, o que sugere que os projetos imobiliários tenham um custo de capital muito menor do que o de determinados projetos em outros setores.

Palavras-chave: fundos de investimento imobiliário, setor imobiliário, modelo CAPM, modelos GARCH. 


\section{INTRODUCCIÓN}

La posibilidad de inversión directa en el sector inmobiliario presenta distintos problemas para un inversionista. Por un lado, al tratarse de compras de activos fijos de alto valor, requieren un monto mínimo de inversión considerable; por ello, la mayoría de familias en el mundo, para quienes la compra de vivienda es tanto gasto en su presupuesto como inversión de largo plazo, deben solicitar un crédito, generalmente hipotecario, para poder acceder a este tipo de inversión. Por otro lado, la posibilidad de diversificación es mínima, al ser los bienes raíces activos indivisibles (Clavijo et al., 2004).

Frente a esto surgen los fondos de inversión inmobiliaria, que permiten a los inversionistas participar de diferentes formas en una inversión conjunta en el sector de finca raíz. Su origen se remonta a los business trust, que aparecieron a mediados del siglo XIX en Estados Unidos y que se transformarían, a partir de los años sesenta, en los Real Estate Investment Trust (REIT), compañías que adquieren y operan activos inmobiliarios $y$ ofrecen a sus clientes un portafolio de posibilidades de inversión diversificadas en estos activos (Bailey, 1966; Asociación Nacional de Instituciones Financieras (ANIF), 2010). La gran mayoría de REIT cotizan en bolsa.

Junto con los REIT, los fondos de capital privado captan recursos del público, con el fin de comprar o desarrollar activos inmobiliarios durante un determinado periodo. En Colombia, su funcionamiento se encuentra amparado en la legislación de carteras colectivas (Decreto 2175 de 2007), que regula la captación de activos para la obtención de resultados económicos (Cámara Colombiana de la Construcción [Camacol], 2008). Otros vehículos de inversión inmobiliaria son las titularizaciones de carteras hipotecarias, que buscan la mitigación de riesgos en los créditos hipotecarios y pueden ser transados en bolsa, y los fideicomisos sobre proyectos inmobiliarios, como hoteles, oficinas y centros comerciales, donde se obtiene una participación en los bienes inmuebles y la rentabilidad depende de los resultados de la explotación de estos.
El presente artículo analiza el comportamiento de cinco fondos inmobiliarios en el 2012 y los compara con la dinámica del mercado accionario en el mismo periodo. Dado que los fondos presentan datos diarios acerca de su valor y valorización, es posible hacer un seguimiento continuo de las series. Adicionalmente, se construye un portafolio con estos fondos, el cual se optimiza de acuerdo con el modelo desarrollado por Markowitz (1952) y Tobin (1958), con el fin de obtener un portafolio óptimo de inversión en el sector inmobiliario. El objetivo final del trabajo es aplicar el modelo de valoración de activos de capital (CAPM, por sus siglas en inglés) desarrollado por Sharpe (1964), en función de estimar la sensibilidad al riesgo no diversificable en el sector inmobiliario. Para esto, se usa la información de los fondos inmobiliarios como proxy del comportamiento del sector y se cuantifica esta sensibilidad a través de la estimación del beta del modelo, tal como lo sugieren Titman y Warga (1986), cuyos trabajos no habían podido ser replicados antes en Colombia debido a la ausencia de estos instrumentos de inversión. Estos resultados son útiles para quienes deben estimar el costo de capital de proyectos en el sector.

La primera parte presentará los fondos escogidos, sus características y comportamiento hasta el 2012, junto con el portafolio construido. La segunda mostrará los modelos utilizados para la estimación del beta y los resultados de las estimaciones. Finalmente, se darán algunas conclusiones.

\section{DESEMPEÑO DE FONDOS INMOBILIARIOS Y CONFORMACIÓN DE PORTAFOLIO}

La aparición de fondos inmobiliarios en Colombia es bastante reciente, comparada con la evolución en el mercado internacional. Solo hasta la mitad de la primera década del siglo XXI pueden registrarse estos instrumentos de inversión ofrecidos por fiduciarias y firmas comisionistas de bolsa. Dentro de sus objetivos de inversión se encuentran, 
en primer lugar, la compra y administración de activos inmobiliarios (donde la rentabilidad se genera por la valorización de estos activos y su arrendamiento), la inversión en proyectos de construcción y la inversión en REIT internacionales. Aunque la redención de estos fondos se pacta en un plazo determinado, es posible seguir la información del valor de la unidad $y$, de esta forma, apreciar su valorización o desvalorización diarias. Esta unidad refleja el valor de redención al cual se podría liquidar el fondo en un momento determinado.

Para el presente trabajo se tomaron cinco fondos inmobiliarios:

a) Fondo Cerrado Inmobiliario Alianza, ofrecido por Fiduciaria Alianza.

b) Fondo de Capital Privado Inmobiliario Ultrabursátiles, de la comisionista Ultrabursátiles S.A.

c) Cartera Colectiva Inmobiliaria Cerrada Interbolsa Inmobiliaria, de la desaparecida comisionista Interbolsa S.A. ${ }^{2}$.

d) Cartera Colectiva Inmobiliaria Inmoval, de la comisionista Correval S.A.

e) Cartera Colectiva Fonval Global Fondos Inmobiliario, de Correval S.A.

Estos fondos deben tener una concentración mínima del $60 \%$ en los siguientes tipos de activos:

a) Bienes inmuebles.

b) Desarrollo de proyectos inmobiliarios.

c) Títulos emitidos en procesos de titularización hipotecaria.

d) Derechos fiduciarios de patrimonios autónomos constituidos con bienes inmuebles.

e) Participaciones en carteras colectivas inmobiliarias extranjeras con características similares a las de Colombia.
La gráfica 1 muestra el comportamiento del valor por unidad de estos fondos, desde el 1 de diciembre de 2011 hasta el 27 de diciembre de 2012. Se muestra el comportamiento de los fondos, comparado con el comportamiento del índice general de la Bolsa de Valores de Colombia (IGBC) y el comportamiento del mercado de renta fija pública, expresado en el valor de los títulos de deuda interna (TES) con vencimiento en julio de 2024. Para hacer posible la comparación, se expresan estas series como índices con valor de 100 al 1 de diciembre de 2011.

El periodo de análisis comienza en diciembre de 2011, ya que en esta fecha empieza a operar el más reciente de los fondos: el Fondo Cerrado Inmobiliario Alianza. Desde entonces, los resultados de estos fondos son positivos, con resultados mixtos, al compararse con el IGBC y el índice de los TES. Mientras que Fonval presenta una rentabilidad muy superior a los indicadores de renta fija y renta variable, la cartera colectiva de Interbolsa tiene el resultado más bajo durante el periodo que precedió la liquidación de esta firma.

La tabla 1 resume las rentabilidades de los fondos, los índices de referencia y la desviación estándar de la rentabilidad diaria, la cual es menor a la del IGBC para todos los fondos; además, muestra una menor volatilidad para el sector inmobiliario consistente con la naturaleza de este tipo de inversión y el comportamiento de los precios del sector en la última década. En efecto, índices como el precio de vivienda usada (IPVU), calculado por el Banco de la República (gráfica 2), o el comportamiento del área aprobada para construcciones (gráfica 3) dan cuenta de una extraordinaria recuperación del sector inmobiliario luego de la gran crisis del 98-99, lo que ha convertido a la finca raíz en una de las inversiones más atractivas en lo que va corrido del siglo XXI, tanto por su rentabilidad como por su baja volatilidad.

2 Esta cartera colectiva estuvo activa hasta enero de 2013, antes de la liquidación definitiva de Interbolsa S.A. 
Gráfica 1

Comportamiento de cinco fondos inmobiliarios en Colombia, IGBC y TES a 2024 (noviembre de 2011-diciembre de 2012)

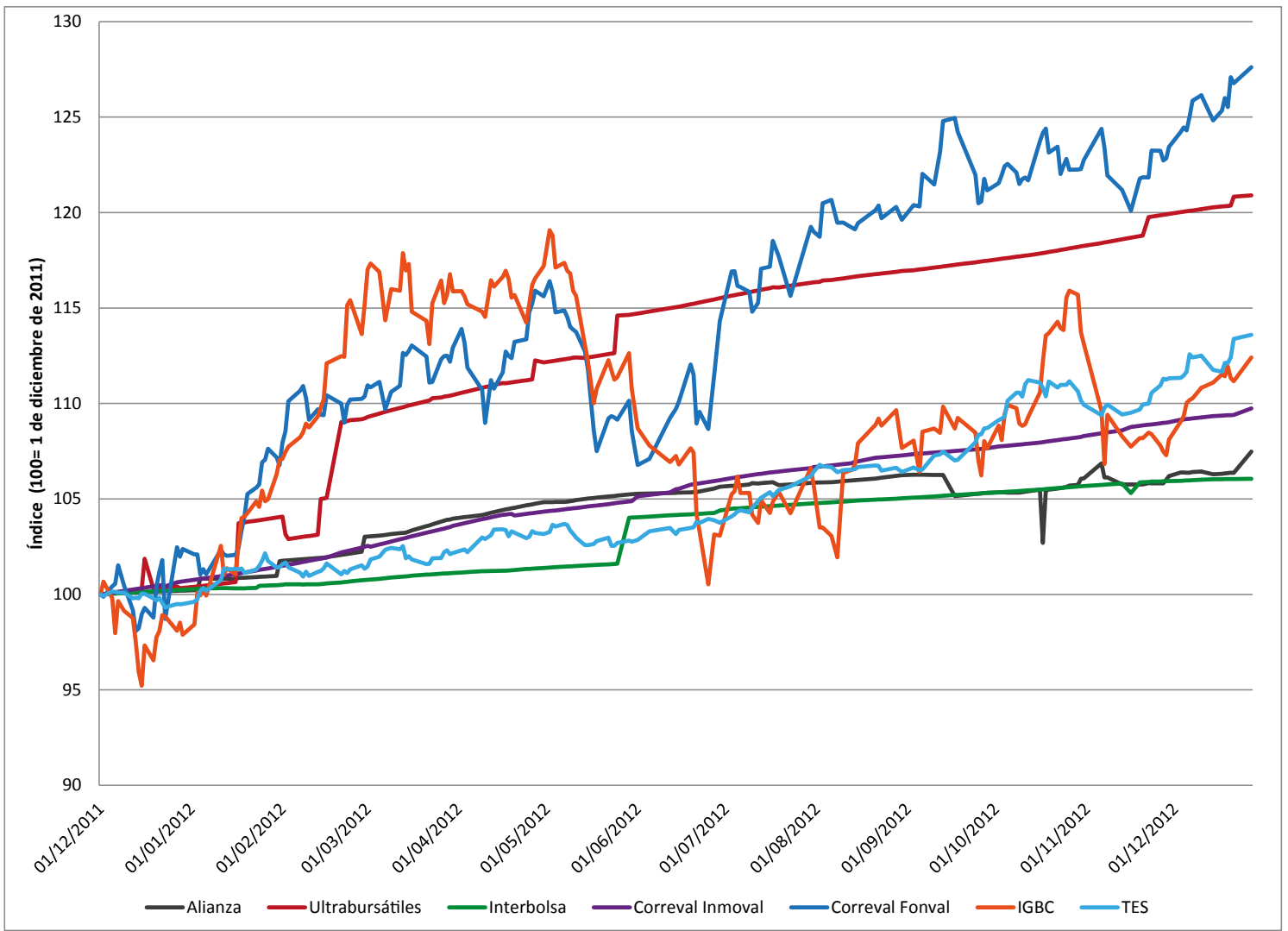

Fuente: Bloomberg (2012).

Tabla 1

Comportamiento de cinco fondos inmobiliarios en Colombia, IGBC y TES a 2024 (noviembre de 2011-diciembre de 2012)

\begin{tabular}{|c|c|c|c|c|c|c|c|}
\hline & Alianza & Ultrabursátiles & Interbolsa & $\begin{array}{c}\text { Correval } \\
\text { Inmoval }\end{array}$ & $\begin{array}{c}\text { Correval } \\
\text { Fonval }\end{array}$ & IGBC & TES \\
\hline Rentabilidad anual en 2012 (\%) & 7,24 & 20,41 & 5,75 & 8,91 & 24,99 & 14,21 & 14,04 \\
\hline $\begin{array}{c}\text { Rentabilidad promedio diaria } \\
\text { en el periodo (\%) }\end{array}$ & 0,0352 & 0,0922 & 0,0284 & 0,0448 & 0,1215 & 0,0622 & 0,0645 \\
\hline $\begin{array}{c}\text { Desviación estándar de } \\
\text { rentabilidad diaria }\end{array}$ & 0,0030 & 0,0042 & 0,0017 & 0,0005 & 0,0093 & 0,0109 & 0,0027 \\
\hline
\end{tabular}

Nota: la rentabilidad es calculada como la variación diaria o anual del valor de la unidad de cada fondo.

Fuente: Bloomberg (2012) y cálculos del autor. 
Gráfica 2

Índice de precios de la vivienda usada (IPVU) en Colombia, 1988-2012

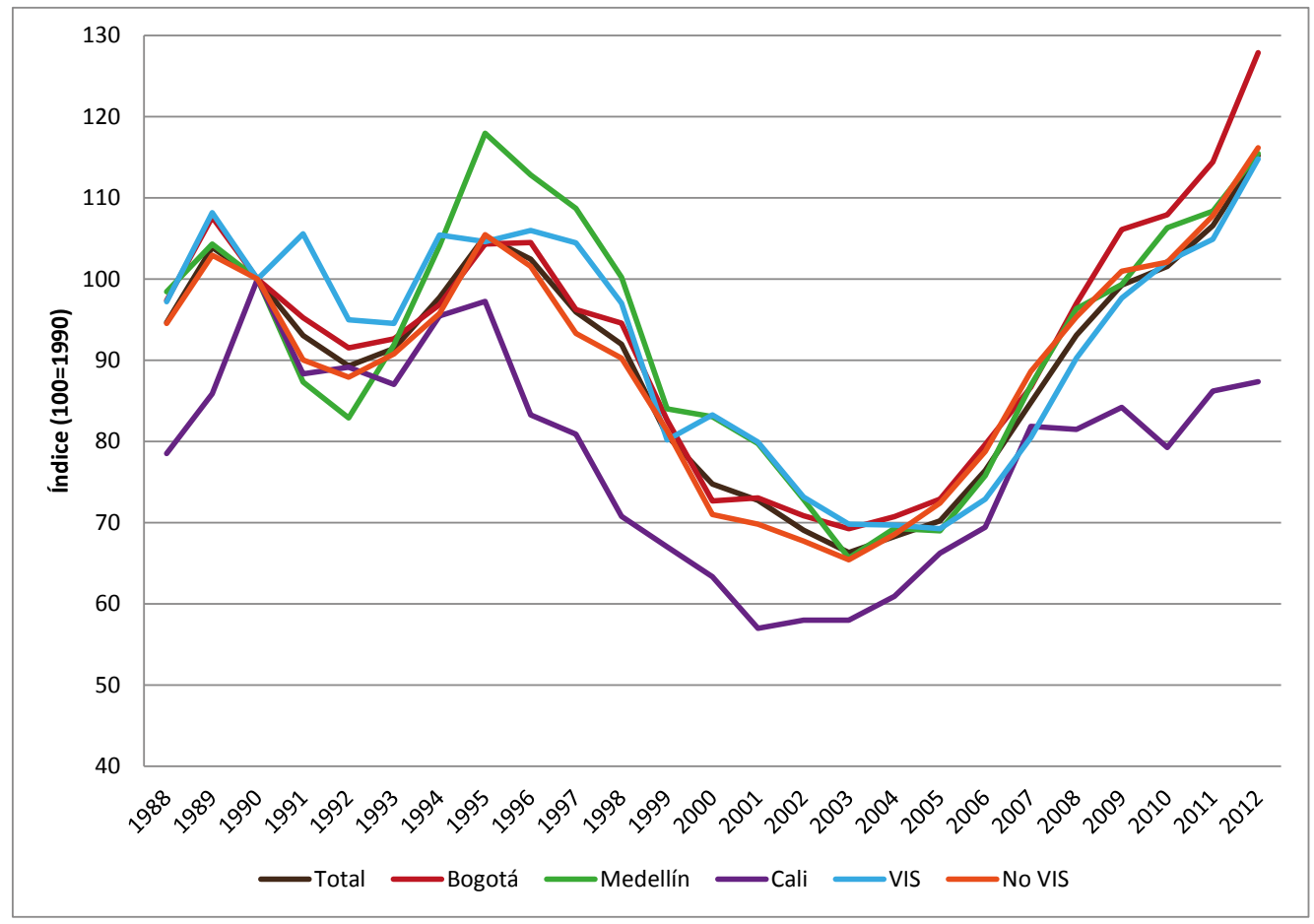

Fuente: Banco de la República (2012).

Gráfica 3

Área aprobada según licencias en Colombia, 1990-2012

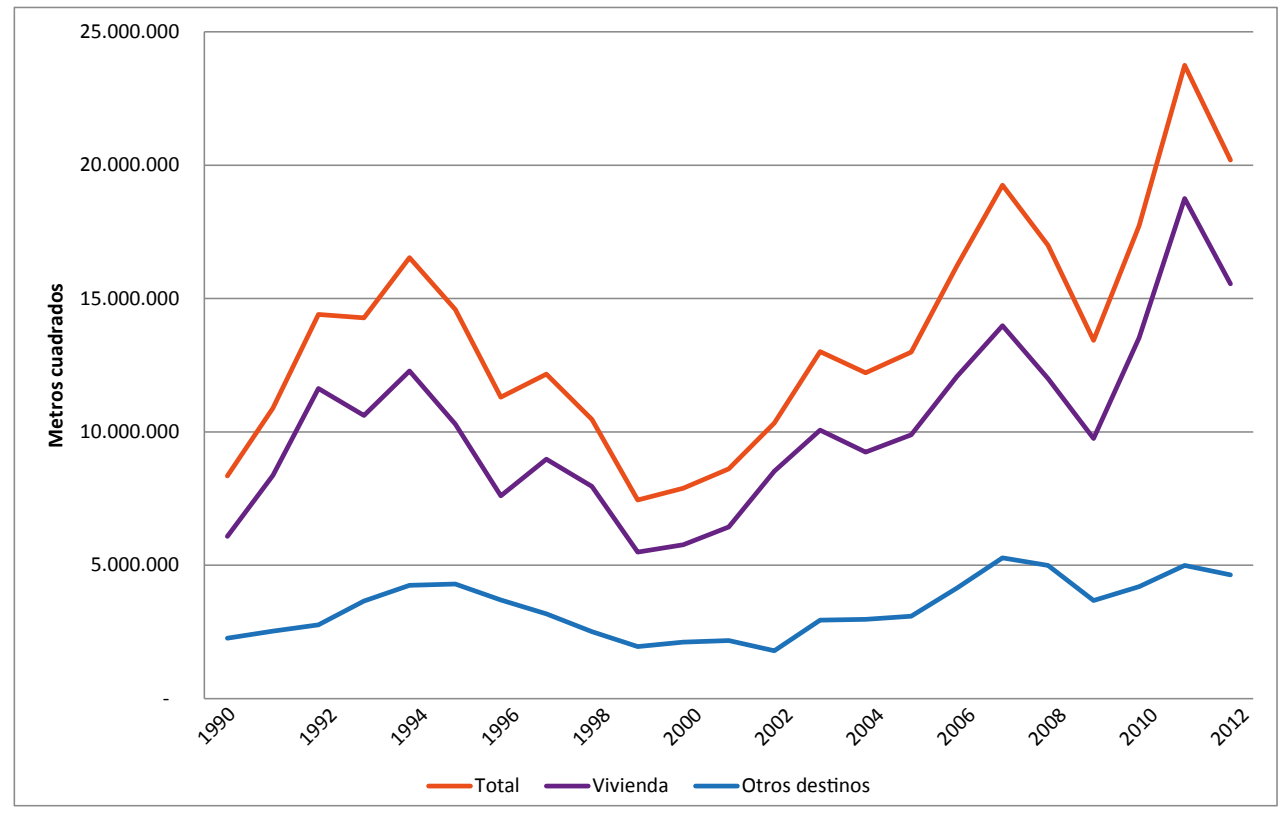

Fuente: Departamento Nacional de Estadística (DANE, 2012). 
Con el fin de realizar la estimación del beta del conjunto de los fondos y estimar su sensibilidad al riesgo no diversificable, se compone un portafolio con las rentabilidades de estos y se optimiza linealmente mediante el modelo propuesto por Markowitz (1952) y extendido por Tobin (1958), quien sugiere la existencia de una óptima combinación de activos al incorporar la posibilidad de inversión de un activo libre de riesgo. Para tal efecto, se usarán como activo libre de riesgo los TES con vencimiento a 2024, los cuales poseen un plazo similar al de los fondos inmobiliarios presentados. De esta forma, se busca encontrar un portafolio óptimo de largo plazo.

La rentabilidad esperada del portafolio $\left(E\left[R_{p}\right]\right)$ está dada por la ecuación [1], donde $W_{i}$ es el porcentaje de participación del i-ésimo fondo inmobiliario en el portafolio y $E(R)$ es la rentabilidad de este i-ésimo fondo para cada uno de los $n$ fondos:

$E\left(R_{p}\right)=\sum_{i=1}^{n} W_{i} E\left(R_{i}\right)$

Por otro lado, el riesgo del portafolio está medido por su desviación estándar $\left(\delta_{p}\right)$ :

$\delta_{p}=\sqrt{\sum_{i}^{n} \sum_{j}^{n} W_{i} W_{j} \delta_{i} \delta_{j} \rho_{i j}}$

Donde eijes el coeficiente de correlación entre el fondo $i$ y el fondo $j$, y $\delta_{i}$ es la desviación estándar del i-ésimo fondo. Luego, el problema de optimización está dado por:

$\operatorname{Max} \frac{E\left(R_{p}\right)-R_{f}}{\delta_{p}}$

s.a.

$0 \leq \mathrm{W}_{\mathrm{i}} \leq 1$

$\sum_{i=1}^{n} W_{i}$

Donde $\left(R_{f}\right)$ es la rentabilidad del activo libre de riesgo, y la suma de las participaciones de cada activo es $100 \%$. De esta forma se maximiza el llamado ratio de Sharpe, que es el resultado de tomar el exceso de rentabilidad del portafolio sobre el activo libre de riesgo y dividirlo entre el riesgo de la inversión medido por la desviación estándar del portafolio. Al aplicar este proceso de optimización a los fondos tratados en este trabajo, el portafolio óptimo presentó las siguientes participaciones $\left(W_{i}\right)$ (tabla 2).

Tabla 2

Participación de los fondos inmobiliarios

\begin{tabular}{|c|c|}
\hline Fondo & Participación (\%) \\
\hline Alianza & 0 \\
\hline Ultrabursátiles & 27,82 \\
\hline Interbolsa & 19,03 \\
\hline Correval Inmoval & 21,29 \\
\hline Correval Fonval & 31,86 \\
\hline
\end{tabular}

Fuente: elaboración del autor.

Puede observarse que el fondo Alianza fue excluido del portafolio óptimo, debido a su baja rentabilidad, combinada con alta correlación positiva con los demás fondos. Este portafolio óptimo tuvo una rentabilidad del $16,59 \%$ E. A. para el 2012. La gráfica 4 muestra el comportamiento del portafolio para el periodo mostrado anteriormente y lo compara con el comportamiento del IGBC, el valor de los TES con vencimiento a 2024 y el activo tomado como libre de riesgo. Se observa cómo este portafolio presenta un mejor desempeño en términos de rentabilidad que los referentes de renta variable y renta fija con los que se compara. Adicionalmente, la gráfica 5 muestra la rentabilidad diaria del portafolio, comparada con la del IGBC para el mismo periodo. Puede apreciarse una menor volatilidad del portafolio frente al mercado de renta variable, lo que presumiblemente indicaría, en el modelo CAPM, un beta para el sector menor a 1. Para comprobar tal presunción, a continuación se estiman diversos modelos, con el fin de calcular el valor de este beta. 
Gráfica 4

Comportamiento del portafolio óptimo de fondos inmobiliarios, IGBC y TES a 2024 (noviembre de 2011-diciembre de 2012)

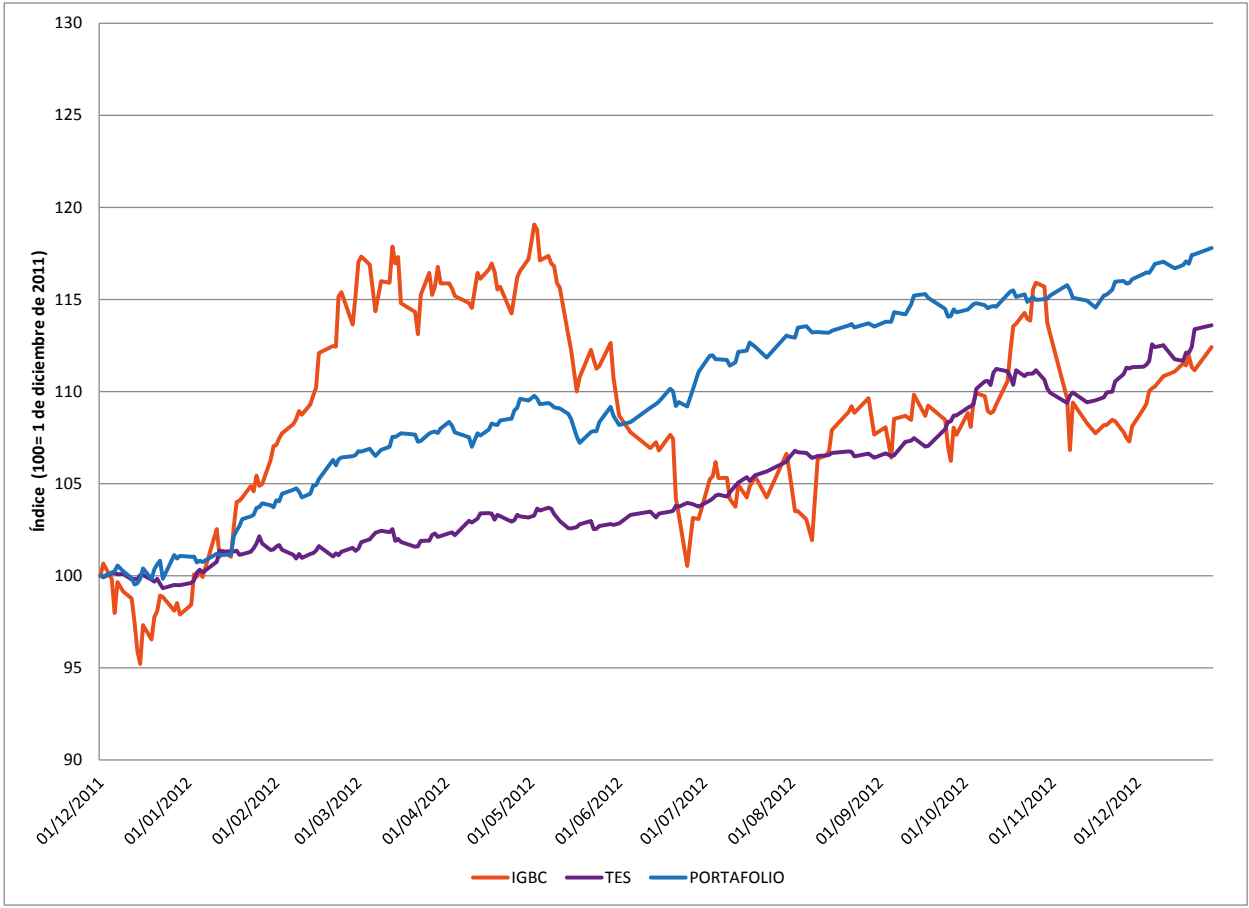

Fuente: Bloomberg (2012) y cálculos del autor.

Gráfica 5

Rentabilidad diaria de portafolio óptimo de fondos inmobiliarios e IGBC (noviembre de 2011-diciembre de 2012)

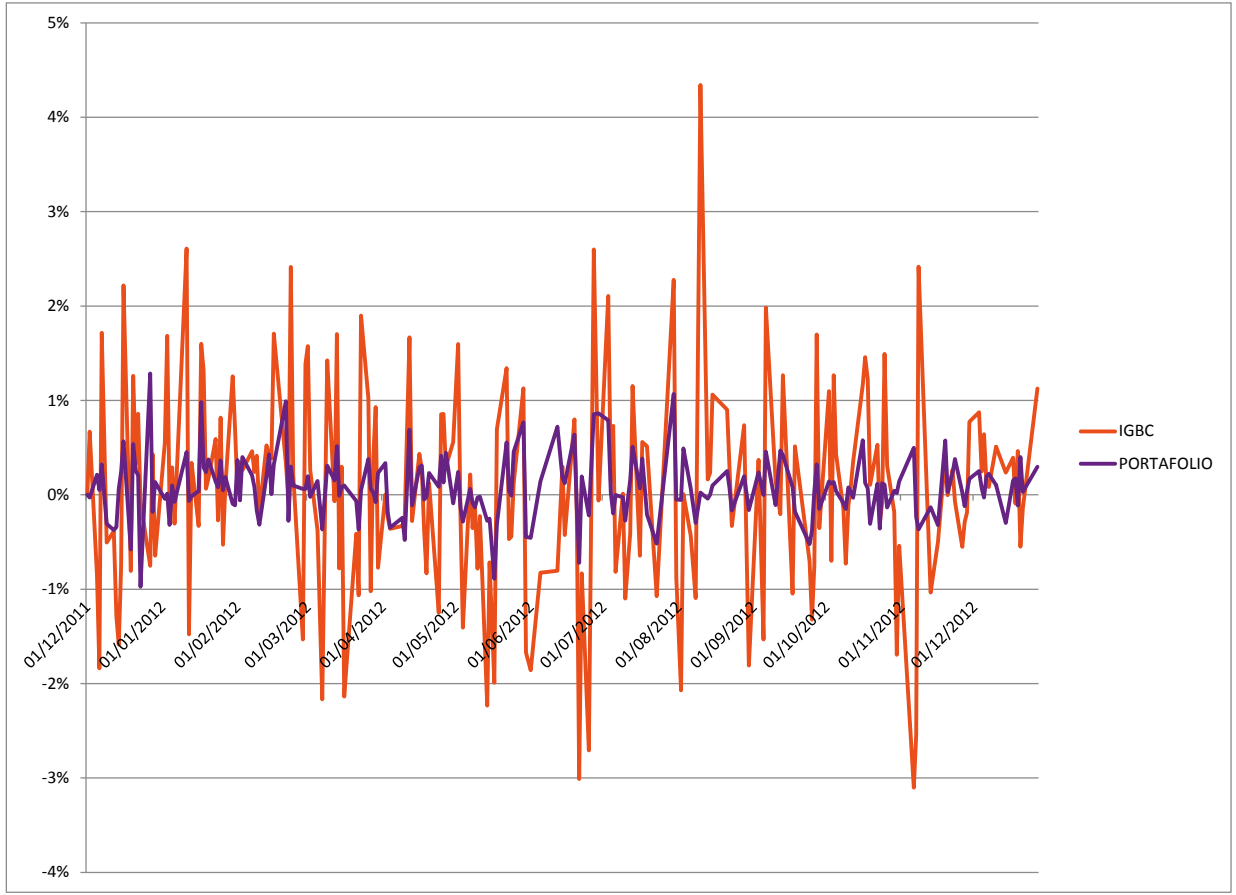

Fuente: Bloomberg (2012) y cálculos del autor. 


\section{ESTIMACIÓN DEL BETA PARA EL SECTOR INMOBILIARIO}

Existen diversas metodologías para estimar el costo de capital de un activo, una empresa o un sector. Dentro de las metodologías cualitativas se tiene, por ejemplo, el proceso de jerarquía analítica (analytical hierarchy process [AHP]), que identifica y organiza factores con el fin de asignar un peso a cada uno en la determinación del riesgo total de la inversión (Palliam, 2005). Sin embargo, la metodología cuantitativa más utilizada es el modelo CAPM, propuesto por Sharpe (1964), que señala una relación de equilibrio entre la rentabilidad esperada de un activo $i\left(E\left[R_{j}\right]\right)$, la rentabilidad de un activo libre de riesgo y la rentabilidad de todos los demás activos de la economía:

$E\left(R_{i}\right)=R_{f}+\beta_{i}\left(R_{m}-R_{f}\right)$

Donde $R_{f}$ es la rentabilidad del activo libre de riesgo y $R_{m}$ es la rentabilidad de todos los demás activos en la economía o rentabilidad de mercado. El término $\left(R_{m}-R_{f}\right)$ es la prima de mercado o lo que renta la economía por encima de la tasa libre de riesgo. A esta prima de mercado se le asocia un riesgo no diversificable o sistemático, por cuanto las variaciones del mercado se encuentran relacionadas con el riesgo de todo el sistema económico (riesgo que no puede ser diversificado). Por último, el $\beta_{i}$ corresponde a la sensibilidad al riesgo no diversificable del activo $i$.

De esta forma, la rentabilidad esperada de un activo debe ser, como mínimo, lo que renta el activo libre de riesgo. A esto se le suma la prima de mercado amplificada o reducida por el valor del beta del activo $(\beta)$. Si el valor de este beta es mayor a 1 , indica un mayor riesgo que el del mercado $y$, por tanto, una mayor rentabilidad esperada. El caso contrario es cuando el beta es menor a 1. La utilidad del modelo CAPM radica en la posibilidad de estimar el costo de capital al momento de realizar valoraciones de empresas, por el método de flujo de caja descontado, o al hacer evaluación de proyectos de inversión nuevos. Hay que aclarar que en este modelo no hay apalancamiento, debido a que la inversión y el rendimiento de los fondos no tienen participación de deuda, es decir, no están apalancados (Vélez-Pareja, 2012).

Para encontrar el valor de las variables $R_{f}$ y $R_{m}$ pueden utilizarse datos de rentabilidades de índices bursátiles o activos específicos. No pasa lo mismo con el beta, que debe ser estimado a partir del comportamiento y la correlación de las variables anteriores. El modelo econométrico más sencillo para estimar este beta consiste en "un proceso generador de rentabilidades en el que se asume que la rentabilidad de mercado es el único factor que determina los cambios sistemáticos en los activos negociados" (Ortas, Moneva y Salvador, 2012, p. 238). De esta forma, se realiza una estimación por mínimos cuadrados ordinarios, donde la variable dependiente es la rentabilidad del activo específico a través del tiempo $\left(R_{t}\right)$, la variable independiente es la rentabilidad de mercado $\left(R_{m, t}\right)$, el beta del modelo CAPM ( $\beta$ ) es el coeficiente asociado a esta última variable y $\varepsilon_{t}$ es el término de error que define el riesgo no sistémico y que se asume como ruido blanco homocedástico:

$R_{t}=\alpha+\beta R_{m . t}+\varepsilon_{t}$

Debido a que la aplicación de este modelo de regresión lineal a series de retornos financieros presenta frecuentemente problemas de autocorrelación serial y heterocedasticidad en el término de error, Tsay (2005), Campbell, Lo y MacKinlay (1996) y Wang (2009) recomiendan el uso de modelos de series de tiempo que incorporen la autocorrelación de las variables analizadas (modelos autorregresivos con promedio móvil y entradas exógenas [ARMAX]) y la heterocedasticidad de los errores (modelos de heterocedasticidad condicional [GARCH), presentados en las ecuaciones 6 y 7 , respectivamente:

$R_{t}=\alpha+\sum_{i=1}^{p} \varphi_{j} R_{t-i}+\beta R_{m, t}+\varepsilon_{t}-\sum_{k=1}^{q} \theta_{k} \varepsilon_{t-k}$

Donde $\alpha, \varphi_{j}$ y $\theta_{k}$ son coeficientes estimados, $\beta$ es el coeficiente asociado al rendimiento 
de mercado (meta del modelo CAPM), $R_{t-i}$ es la rentabilidad del activo en el momento $t-i, R_{m, t}$ es la rentabilidad de mercado en el momento $t$ y $\varepsilon_{t-k}$ es el ruido blanco en el momento $t-k$.

En el caso de los modelos GARCH, la varianza esperada del activo $\left(\sigma^{2}\right)$ está dada por:

$\sigma^{2}=\omega+\sum_{i=1}^{m} \gamma_{i} \varepsilon_{t-i}^{2}+\sum_{j=1}^{s} \rho_{j} \sigma_{t-j}^{2}$

Donde $\omega, \gamma_{j}$ y $\rho_{j}$ son coeficientes estimados de las variables y $\sigma_{t-j}^{2}$ es la varianza esperada en el momento $t-j$.

Sin embargo, pueden encontrarse otros métodos de estimación, como el que usan Shalit y Yitzhaki (2002), con un modelo basado en el estimador de Gini. Además, desde el trabajo de Blume (1971) se ha prestado especial atención al tema de la inestabilidad de los betas y a la estimación de su cambio a través del tiempo. Por esto, los trabajos recientes en todo el mundo que buscan estimar betas para distintos activos han desarrollado una serie de modelos que intentan incorporar un beta dinámico. Por ejemplo, Lin, Chen y Boot (1992) usan modelos variable-media-respuesta (VMR) para calcular betas de varias acciones de la Bolsa de Valores de Nueva York; Das y Goshal (2010) utilizan filtros de Kalman en el proceso de estimación de betas dinámicos de índices de la Bolsa Nacional de Valores de India; mientras que Ortas, Moneva y Salvador (2012) emplean modelos espacio-estado para carteras sectoriales de la Bolsa de Madrid.

Dado que la ventana de observación del presente trabajo es de poco más de un año, se conservará el supuesto de beta constante a través del tiempo, y se estimará el beta para los distintos fondos y el portafolio construido mediante los modelos descritos en las ecuaciones [5], [6] y [7]. Para ello, $R_{t}$ estará dado por la rentabilidad diaria de cada fondo y del portafolio y $R_{m, t}$ por el índice bursátil de la Bolsa de Valores de Colombia. Antes de aplicar estos métodos de estimación se comprobó la estacionariedad de las variables mediante la prueba de Phillips-Perron para los datos de rentabilidades diarias del IGBC, de los cinco fondos y del portafolio, entre el 1 de diciembre de 2011 y el 27 de diciembre de 2012. Luego se estimó el beta mediante modelos de mínimos cuadrados ordinarios y modelos ARMAX. Se revisó en estos últimos el comportamiento de los errores y se encontró evidencia de heterocedasticidad en todos ellos al usar la prueba Breusch-Pagan. Por lo anterior, se estiman finalmente modelos GARCH. La tabla 3 resume los resultados de las estimaciones según el modelo utilizado.

Tabla 3

Resultados de estimaciones del beta para cada fondo inmobiliario y portafolio construido

\begin{tabular}{|c|c|c|c|}
\hline Fondo & MCO. & ARMAX & GARCH \\
\hline Alianza & 0,00998 & 0,0108397 & 0,005974 \\
\hline Ultrabúrsatiles & $0,0459445^{*}$ & 0,049366 & 0,04552 \\
\hline Interbolsa & 0,0110596 & 0,061614 & 0,05466 \\
\hline Correval Inmoval & $-0,0003632$ & 0,0007734 & $-0,0002545$ \\
\hline Correval Fonval & $0,376948^{* * *}$ & $0,3647539^{* * *}$ & $0,3610236^{* * *}$ \\
\hline Portafolio & $0,134886^{* * *}$ & $0,1380427^{* * *}$ & $0,1190921^{* * *}$ \\
\hline
\end{tabular}

*** Significativa al $99 \%$ de confianza.

** Significativa al $95 \%$ de confianza.

* Significativa al $90 \%$ de confianza.

Fuente: cálculos del autor. 
Como puede observarse, a excepción del fondo Fonval, los coeficientes no son significativos al $95 \%$ de confianza. Sin embargo, al ser combinados en el portafolio presentado, la estimación del beta sí presenta una confianza superior al $99 \%$ de que este coeficiente es diferente de 0 . El valor estimado es bastante bajo $(0,134886$ para el modelo de mínimos cuadrados ordinarios [MCO], 0,1380427 para el modelo ARMAX y 0,1190921 para el modelo $\mathrm{GARCH}$ ), comparado con los betas estimados en otros trabajos para sectores diferentes. Buenaventura, Gómez y Ortiz (2010), por ejemplo, calcularon el beta para distintas firmas que cotizan en la Bolsa de Valores de Colombia y encontraron coeficientes positivos que fluctúan en el rango de 0,12 a 1,6; mientras que Ramírez y Serna (2012) calculan el modelo CAPM tradicional para diferentes acciones de esta misma bolsa, con estimaciones de betas significativos positivos entre 0,003 y 1,44. De esta forma, el beta del sector inmobiliario estimado en este trabajo, a partir del comportamiento del portafolio constituido por fondos colombianos dedicados a la inversión en este sector, presenta uno de los menores betas sectoriales $y$, por tanto, uno de los menores costos de capital.

Finalmente, la tabla 4 presenta los intervalos de confianza, con un $95 \%$ para el valor del beta en los tres modelos del portafolio inmobiliario construido. En el caso del modelo ARMAX, se usaron los rezagos 1 y 3 de la variable independiente y los rezagos 3 y 5 en el componente de media móvil. Para el modelo GARCH, se usó el rezago 2 en el componente $A R C H$ y los rezagos 1 y 2 en el componente GARCH. Como puede observarse, los

Tabla 4

Estimación de betas: intervalos de confianza para modelo con portafolio (95\% de confianza)

\begin{tabular}{|c|c|c|}
\hline Modelo & Límite superior & Límite inferior \\
\hline MCO & 0,1715326 & 0,0982393 \\
\hline ARMAX & 0,1652748 & 0,1108107 \\
\hline GARCH & 0,1464327 & 0,0917514 \\
\hline
\end{tabular}

Fuente: cálculos del autor. valores estimados del beta dentro de los intervalos de confianza fluctúan entre 0,09 y 0,18, lo cual muestra un beta sectorial muy bajo debido a la alta y sostenida rentabilidad que el sector inmobiliario ha tenido en los últimos años, producto de las altas valorizaciones en precios de inmuebles en todo el país (en contraposición a la coyuntura internacional) y a la buena dinámica del sector constructor.

\section{CONCLUSIONES}

A través del modelo de optimización de Markowitz (1952) y Tobin (1958) puede obtenerse un portafolio de fondos óptimo con una rentabilidad anual del $16,59 \%$. Ello permite evidenciar cómo estos nuevos instrumentos de inversión constituyen una atractiva alternativa de posicionamiento de recursos para los inversionistas y un canal importante de financiamiento de un dinámico sector inmobiliario.

Para trabajos posteriores que usen el resultado de esta investigación en ejercicios de valoración de empresas inmobiliarias o evaluación de proyectos inmobiliarios, se recomienda tomar los resultados del modelo GARCH estimado para el portafolio, por cuanto muestra ser el de mejor estimación, según el criterio de información de Akaike. Por tanto, el beta sectorial inmobiliario puede tomarse entre 0,15 y 0,09 , lo cual indica una baja sensibilidad del portafolio (y del sector inmobiliario) al riesgo de mercado. Al compararlo con estimaciones de betas para otros sectores en Colombia, el inmobiliario presentaría la menor sensibilidad al riesgo no diversificable de todos. El uso de un beta dentro de este rango tiene como consecuencia tasas de descuento y un costo de capital más bajo para proyectos de inversión en este sector que para los demás sectores de la economía.

Es importante resaltar que estos resultados reflejan un beta para un periodo alcista del ciclo inmobiliario. Posteriores trabajos pueden explorar, dentro de este u otros sectores, una dinámica de betas cambiantes relacionados con la teoría de ciclos, ampliando el periodo analizado a medida que crezcan las alternativas de inversión inmobiliaria de portafolio en Colombia. 


\section{REFERENCIAS}

1. Asociación Nacional de Instituciones Financieras (ANIF) (2010). Mercado inmobiliario internacional: el caso de los REITS. En Enfoque Mercado de Capitales (núm. 42). Bogotá: Autor.

2. Bailey, E. (1966). Real Estate Investment Trusts: an appraisal. Financial Analysts Journal, 22 (3), 107-114.

3. Blume, M. (1971). On the assessment of risk. The Journal of Finance, 26(1), 1-10.

4. Buenaventura G., Gómez, C. y Ortiz, J. (2010). Aplicación de las teorías de la firma: operacionalización del CAPM para empresas de Colombia y latinoamericanas. Bogotá: Universidad ICESI. Recuperado de http://www.icesi.edu.co/departamentos/finanzas_contabilidad/images/working_papers/capm.pdf

5. Cámara Colombiana de la Construcción (Camacol) (2008). Alternativas para financiar la construcción. Informe Económico, 4. Recuperado de http://camacol.co/sites/default/files/secciones_internas/ EE_Coy20080503043954.pdf

6. Campbell, J., Lo, A. y MacKinlay, A. (1996). The econometrics of financial markets. Nueva Jersey: Princeton University Press.

7. Clavijo, S. Janna, M. y Muñoz, S. (2004). La vivienda en Colombia: sus determinantes socioeconómicos y financieros. Borradores de Economía, 300. Recuperado de http://www.banrep.gov.co/docum/ftp/borra300.pdf

8. Departamento Nacional de Estadística (DANE) (2012). Estadísticas de licencias de construcción. Recuperado de http://www.dane.gov.co/index.php/construccion-alias/estadisticas -de-edificacion-de-licencias-de-construccion-elic

9. Das, A. y Goshal, T. (2010). Market risk beta estimation using adaptative Kalman filter. International Journal of Engineering Science and Technology, 2(6), 1923-1934.

10. Lin, W., Chen, Y. y Boot, J. (1992). The dynamic and stochastic instability of betas: implications for forecasting stock returns. Journal of Forecasting, 11, 517-541.

11. Markowitz, H. (1952). Portfolio selection. The Journal of Finance, 7(1), 77-91.

12. Ortas, E., Moneva, J. y Salvador, M. (2012). Dinámica del coeficiente beta asociado a las carteras de inversión. Revista Española de Financiación y Contabilidad, 41 (154), 233-261.

13. Palliam, R (2005). Estimating the cost of capital: considerations for small business. The Journal of Risk Finance, 6(4), 335-340.

14. Presidencia de la República de Colombia (2007, 12 de junio). Decreto 2175 de 2007, por el cual se regula la administración y gestión de las carteras colectivas. Diario Oficial 46657.

15. Ramírez, A. y Serna, M. (2012). Validación empírica del modelo CAPM para Colombia 2003-2010. Ecos de Economía, 34, 49-74

16. Shalit, H. y Yitzhaki, S. (2002). Estimating beta. Review of Quantitative Finance and Accounting, 18, 95-118.

17. Sharpe, W. (1964). Capital assets prices: a theory of market equilibrium under conditions of risk. The Journal of Finance, 19(3), 425-442.

18. Titman, S. y Warga, A. (1986). Risk and performance of real estate investment trust: a multiple index approach. AREUEA Journal, 14, 414-431.

19. Tobin, J. (1958). Liquidity preference as behavior towards risk. The Review of Economic Studies, 67, 65-86.

20. Tsay, R. (2005). Analysis of financial time series (2. ${ }^{\mathrm{a}}$ ed.). Nueva Jersey: Wiley-Interscience. 
21. Vélez-Pareja, I. (2011). Estimación de betas y relación entre las betas apalancadas y el coste de capital. Análisis Financiero, 116, 6-13

22. Wang, P. (2009). Financial econometrics (2. ${ }^{a}$ ed.). Nueva York: Routledge. 
